\title{
Structure of magnetic fields in spiral galaxies
}

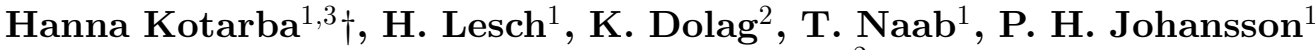 \\ and F. A. Stasyszyn ${ }^{2}$ \\ ${ }^{1}$ University Observatory Munich, Scheinerstr. 1, D-81679 Munich, Germany \\ email: kotarba@usm.lmu.de \\ ${ }^{2}$ MPI for Astrophysics, Karl-Schwarzschild-Str. 1, D-85741 Garching, Germany \\ ${ }^{3}$ MPI for Extraterrestrial Physics, Giessenbachstrasse, D-85748 Garching, Germany
}

\begin{abstract}
We present a set of global, self-consistent $N$-body/SPH simulations of the dynamic evolution of galactic discs with gas and including magnetic fields. We have implemented a description to follow the ideal induction equation in the SPH part of the code VINE. Results from a direct implementation of the field equations are compared to a representation by Euler potentials, which pose a $\boldsymbol{\nabla} \cdot \boldsymbol{B}$-free description, a constraint not fulfilled for the direct implementation. All simulations are compared to an implementation of magnetic fields in the code GADGET. Starting with a homogeneous field we find a tight connection of the magnetic field structure to the density pattern of the galaxy in our simulations, with the magnetic field lines being aligned with the developing spiral pattern of the gas. Our simulations clearly show the importance of non-axisymmetry of the dynamic pattern for the evolution of the magnetic field.
\end{abstract}

Keywords. Methods: n-body simulations - magnetic fields - galaxies: evolution - galaxies: spiral

\section{Numerical Methods and Setup}

Hydrodynamics including the ideal induction equation are treated by the SPH method implemented within the OpenMP parallel $N$-body code VINE (Wetzstein et al. 2008, Nelson et al. 2008). We have also implemented a description of magnetic fields using the Euler potentials, which are free of $\boldsymbol{\nabla} \cdot \boldsymbol{B}$ per construction $\left(\boldsymbol{B}=\boldsymbol{\nabla} \alpha_{E} \times \boldsymbol{\nabla} \beta_{E}\right.$, where $\alpha_{E}$ and $\beta_{E}$ are scalar potentials).

A similar treatment of magnetic fields is implemented in the MPI parallel $N$-body/SPH code Gadget (Springel 2005, Dolag \& Stasyszyn 2008) which includes also cleaning methods for $\boldsymbol{\nabla} \cdot \boldsymbol{B}$. The initial conditions for our Milky Way like galaxy are realized using the method described by Springel et al. (2005) which is based on Hernquist (1993). The galaxy consists of an exponential stellar disc and a flat extended gas disc, a stellar bulge and a dark matter halo of collisionless particles. We adopt an isothermal equation of state with a fixed sound speed of $\approx 15 \mathrm{~km} / \mathrm{s}$.

\section{Results}

The magnetic field strength pattern is tightly connected to the density pattern of the gas in our simulated galaxy for all implementations (figure 1). Using the direct implementation of the induction equation, the largest magnetic field strengths are reached in the center (not shown), whereas in the Euler implementation the largest values are reached in the spiral arms. The morphology shows great similarity for both implementations. Using Euler potentials, the magnetic field gets amplified by one order of magnitude during the simulation, which is still one to two orders of magnitude below the amplification for the

$\dagger$ Present address: University Observatory, Scheinerstr. 1, D-81679 Munich, Germany 


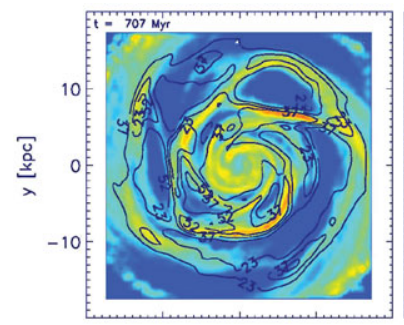

$\times[\mathrm{kpc}]$

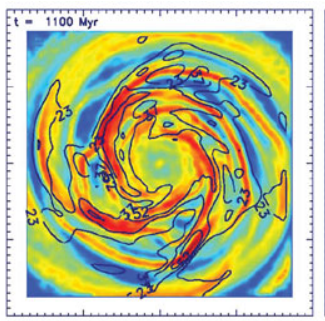

$x[\mathrm{kpc}]$

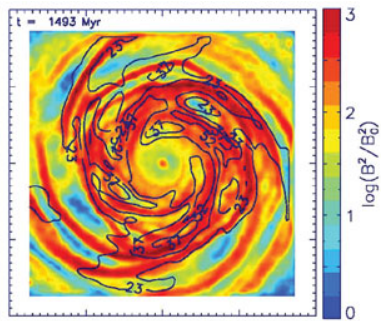

$\times[\mathrm{kpc}]$

Figure 1. Face-on magnetic field energy and gas density as a function of time for a simulation performed with VINE using the Euler potentials. The colors correspond to the magnetic field energy $B^{2} / 8 \pi$ on a logarithmic scale, normalized to the initial value of $\frac{1}{8 \pi} \cdot 10^{-18} \mathrm{erg} \mathrm{cm}^{-3}$. The contour lines indicate physical densities of 23,37 and $52 M_{\odot} \mathrm{pc}^{-3}$, respectively.
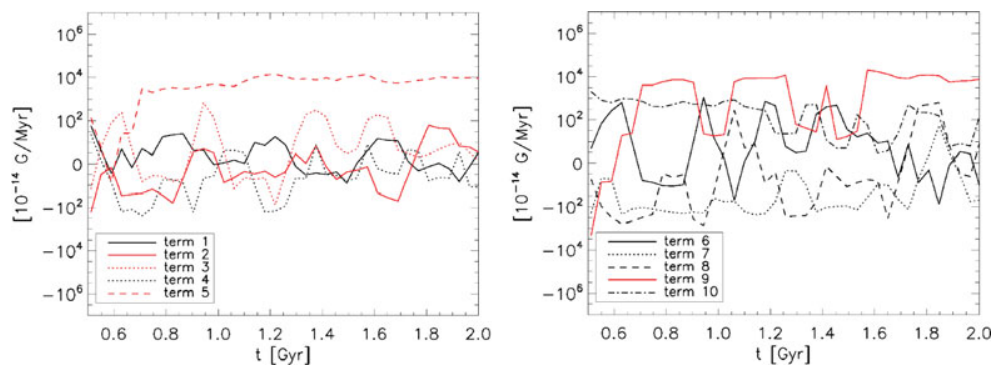

Figure 2. Values of terms responsible for the evolution of the radial (left graphic) and toroidal magnetic field component (right graphic) as a function of time for a GADGET simulation using Euler potentials. Positive values imply amplification, and negative attenuation of the corresponding $\boldsymbol{B}$-component. Non-axisymmetric terms are shown in red.

direct implementation. In all cases, the growth of $\boldsymbol{\nabla} \cdot \boldsymbol{B}$ correlates with the growth of the total magnetic field, i.e. the higher the divergence, the stronger the growth of the magnetic field. Applying smoothing of $\boldsymbol{B}$ lowers the divergence (Dolag \& Stasyszyn 2008) and lowers also the field amplification, leading to an amplification of the total field similar to that using the Euler potentials (not shown). Thus, for an ideal treatment, Euler potentials are a good method to follow the evolution of magnetic fields in spiral galaxies.

Dropping all dependencies on $z$, the equations for the temporal evolution of the radial and toroidal magnetic field depend on five terms each. Figure 2 displays the values of the terms responsible for the evolution of the radial (left graphic) and the toroidal magnetic field component (right graphic) as a function of time for a GADGET simulation using Euler potentials. Terms depending on azimuthal derivatives are shown in red. Although azimuthal derivatives are comparatively small, these terms have the largest values, thus showing that even small deviations from axial symmetry are of crucial importance for the evolution of the magnetic field.

\section{References}

Dolag, K. \& Stasyszyn, F. A. 2008, ArXiv e-prints arXiv:0807.3553

Wetzstein, M., Nelson, A. F., Naab, T., \& Burkert A. 2008, ArXiv e-prints arXiv:0802.4245

Nelson, A. F., Wetzstein, M., \& Naab, T. 2008, ArXiv e-prints arXiv:0802.4253

Springel, V., Di Matteo, T., \& Hernquist, L. 2005, MNRAS 361, 776

Hernquist, L. 1993, ApJ 86, 389

Springel, V. 2005, MNRAS 364, 1105 